\title{
Using a Foundational Ontology for Reengineering a Software Enterprise Ontology
}

\author{
Monalessa Perini Barcellos ${ }^{1,2}$, Ricardo de Almeida Falbo ${ }^{1}$ \\ ${ }^{1}$ Ontology and Conceptual Modeling Research Group (NEMO), Federal University of \\ Espírito Santo - Brazil \\ ${ }^{2}$ COPPE, Federal University of Rio de Janeiro - Brazil \\ \{monalessa, falbo\}@inf.ufes.br
}

\begin{abstract}
The knowledge about software organizations is considerably relevant to software engineers. The use of a common vocabulary for representing the useful knowledge about software organizations involved in software projects is important for several reasons, such as to support knowledge reuse and to allow communication and interoperability between tools. Domain ontologies can be used to define a common vocabulary for sharing and reuse of knowledge about some domain. Foundational ontologies can be used for evaluating and redesigning domain ontologies, giving to these real-world semantics. This paper presents an evaluating of a Software Enterprise Ontology that was reengineered using the Unified Foundation Ontology (UFO) as basis.
\end{abstract}

Keywords: Foundational Ontology, Enterprise Ontology, Ontology Reengineering.

\section{Introduction}

Software engineering is a knowledge-intensive activity and there are many types of knowledge that are useful for software engineers. One of the main obstacles for capturing, searching and reusing this knowledge is the lack of a common conceptualizations, what can be provided by ontologies.

In context of Software Engineering Environments, ontologies have been used, among others, for building Domain Oriented Software Development Environments (DOSDEs) [1]. DOSDEs support software engineers in their tasks by providing useful knowledge during the software development process.

By building several DOSDEs for different domains, researches have noticed that other kinds of knowledge beyond domain knowledge could also be useful for software projects, mainly knowledge about the organizations involved in this context. Based on that, Villela et al. [2] defined a Software Enterprise Ontology (SEO) that was used as basis for building what they call Enterprise Oriented Software Development Environments (EOSDEs). 
During the development of a research work considering high maturity aspects on software organizations supported by EOSDEs (characterized by the highest levels of maturity models like CMMI), we envision the need for building a measurement ontology. Since ideally domain ontologies should be grounded in foundational ontologies [3, 4], we decided to develop our measurement ontology taking as basis the Unified Foundational Ontology (UFO) [3, 5]. UFO has been used to evaluate, redesign and integrate (meta) models of conceptual modeling languages, as well as to evaluate, re-design and give real-world semantics to domain ontologies [5]. Besides, we noticed that in order to talk about measurement in high maturity software organizations, it was also necessary to use concepts regarding software organizations. Then we decided to use some concepts of SEO [2]. However, we had several problems, mainly due to implicit ontological commitments, as well as to real-world situations that were not addressed by SEO. Before integrating the ontologies, we decided to carry out an evaluation and reengineering of SEO by mapping its concepts to UFO. This allowed us to solve conceptual problems, making SEO more truthful to the domain it represents, and making explicit some ontological commitments that were implicit.

This paper presents the SEO evaluation and reengineering and it is organized as follows: Section 2 presents a brief discussion about ontologies, and presents relevant parts of UFO to this work and the fragment of SEO considered; in Section 3, we discuss the evaluation and reengineering of SEO; Section 4 presents related works; and in Section 5, we conclude presenting the final considerations of the work done.

\section{Ontologies}

In the context of Philosophy, ontology is a particular system of categories accounting for a certain vision of the world, independent of a particular language. Otherwise, for Computer Science communities, ontologies refer to an engineering artifact, constituted by a specific vocabulary used to describe a certain reality and by a set of explicit assumptions regarding the intended meaning of the vocabulary words [4].

In the context of the use of ontologies in Computer Science, Guarino [4] states that computational ontologies mix philosophical, cognitive and linguistic aspects, and ignoring their interdisciplinary nature makes the ontologies less useful. To capture this, Guarino says that, ideally, domain ontologies should be built based on Foundational Ontologies. Foundational ontologies are theoretically well-founded and domain- independent systems of categories, which describe very general concepts like object, event, action etc [5]. Due to its soundness, foundational ontologies can be used to improve the quality of conceptual models, including domain ontologies [8].

UFO $[3,5]$ is a foundational ontology that has been developed based on a number of theories from Formal Ontology, Philosophical Logics, Philosophy of Language, Linguistics and Cognitive Psychology. It is composed by three main parts. UFO-A is an ontology of endurants, and it is the core of UFO. A fundamental distinction in UFO-A is between Particulars (Individuals) and Universals (Types). Particulars are entities that exist in reality possessing a unique identity, while Universals are patterns of features, which can be realized in a number of different particulars [3]. UFO-B is 
an ontology of perdurants (events). The main distinction between perdurants and endurants is that endurants are wholly present or not, while perdurants happen in time [5]. UFO-C is an ontology of social entities (both endurants and perdurants) built on the top of UFO-A and UFO-B. One of its main distinctions is between agents and objects. Agents are capable of performing actions with some intention, while objects only participate in events [5].

For the purpose of this paper, concepts from parts A and C of UFO are more important, since the analyzed fragment of SEO does not talk about events. Due to space limitations, it is impossible to discuss here all the distinctions made in those parts of UFO. So, Figure 1 presents some concepts that are important for this paper. The concepts that are directly used here are shown shaded in grey. It is worthwhile to point out that, since we used UFO concepts to ground concepts of a domain ontology, concepts related to Universals were more important. Following some of the concepts shown in Figure 1 are described.

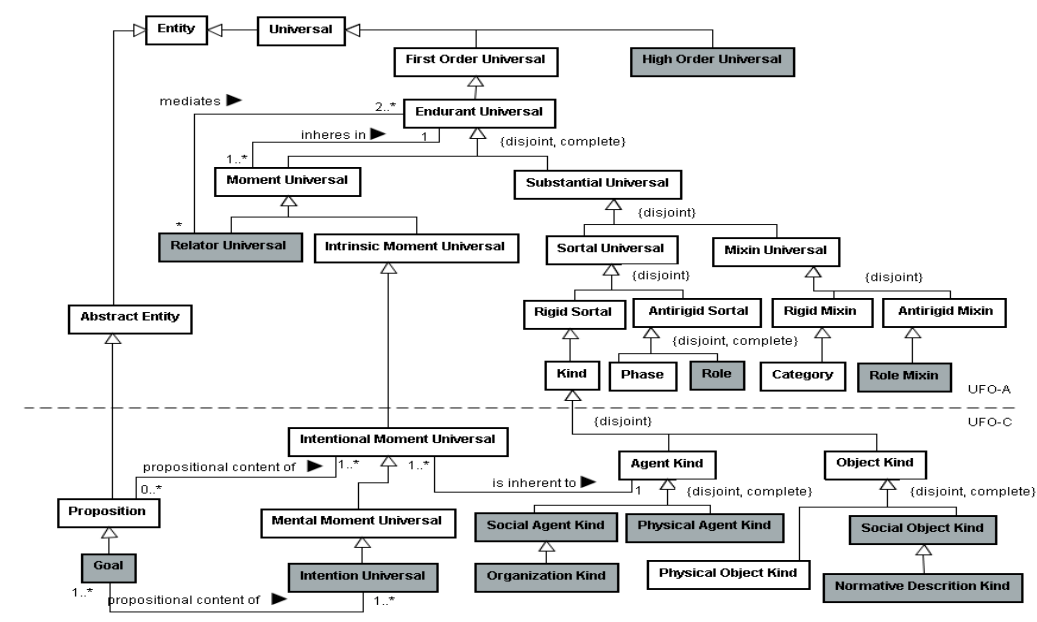

Fig. 1. An UFO Fragment.

- First Order Universal (UFO-A): universals whose instances are particulars.

- High Order Universal (UFO-A): universals whose instances are universals.

- Kind (UFO-A): a substance sortal ${ }^{1}$ universal that supplies a principle of identity for its instances (rigid sortals) [3].

- Role (UFO-A): a possible role that a substance sortal can play along its history. An entity plays a role in a certain context, demarcated by its relations with other entities [3].

- Role Mixin (UFO-A): Anti-rigid mixin ${ }^{2}$ that represents abstractions of common properties of roles [3].

${ }^{1}$ Substantials are entities that persist in time, keeping their identity. Substantial universals are patterns of features that can be realized in a number of different substantials. Some of them are sortal (sortal universals), thus providing a principle of individualization, persistence and identity. Others are merely characterizing (said mixin universals) [3]. 
- Intrinsic Moment Universal (UFO-A): a moment universal ${ }^{3}$ that is dependent on a single universal.

- Relator Universal (UFO-A): a moment universal that is dependent on a plurality of universals. It is a mediating entity, i.e., a moment universal with the power of connecting other universals. It is existentially dependent of the endurant universals that it mediates ${ }^{4}[3]$.

- Agent Kind (UFO-C): An agentive substantial universal whose instances (agents) are capable to refer to possible situations of reality and that can bear special kinds of moments, named intentional moments. Only agents can perform actions. In other words, intentions cause the agent to perform actions [5]. Agent kinds can be physical (e.g., Person) or social (e.g., Organization, Society).

- Object Kind (UFO-C): Non-agentive substantial universal. Its instances (objects) do not act. They can only participate in actions. Object kinds can also be further categorized into physical (e.g., Book) and social (e.g., Language) [5].

- Normative Description Kind (UFO-C): a social object kind whose instances define one or more rules / norms recognized by at least one social agent and that can define nominal universals [5].

- Intention Universal (UFO-C): a type of intentionality designating "intending something" [5].

- Goal $(U F O-C)$ : The propositional content of an intention [5].

In this paper, the distinctions made in UFO are shown in the concepts of the SEO as stereotypes, indicating that they are subtypes of concepts of UFO, in an approach analogous to the one defined in [3]. As pointed out by Guizzardi [3], the ontological interpretation of a UML class is of an endurant universal. Thus for simplicity, the term universal (or kind, depending on the situation) was omitted from the corresponding stereotypes. For example, the stereotype $<<$ social object $>>$ designates the UFO's concept of Social Object Kind, shown in Figure 1. When a concept is not stereotyped then it presents the same stereotype of its super-type in the model.

The Software Enterprise Ontology (SEO) considered in this paper was developed by Villela et al. [2] to establish a common vocabulary for software engineers to talk about the organizations involved in software projects. Figure 2 presents the fragment of SEO considered in this paper. According to [2], an Organization is an organized group of people working together for the fulfillment of a mission. Mission is the organization's purpose in a social or economic system. An organization is divided into Functions, Organization Units and Committees. Organization Units can be structured by Positions. An Agent represents a profile that allows the organization to accomplish its mission and it can be a function or a position. A Committee is a group

${ }^{2}$ Mixins are dispersive universals, covering many concepts with different principles of identity. Anti-rigid mixins are mixins of witch patterns of features does not apply necessarily to all its instances [3].

3 The word Moment in UFO-A is derived from the German term Momente and it bears no relation to the notion of time instant. It is related to the ways things are. An important feature that characterizes all moments is that they can only exist in other individuals. Thus, moment universals can only exist in other universals [3].

${ }^{4} \mathrm{~A}$ mediation is a formal relation that takes place between a relator universal and the endurant universals it mediates [3]. 
of people with a specific goal, which usually works together for a period of time until a specific goal is achieved. Organizations have Participations on Projects. A Business Agreement is an agreement between two or more organizations which establishes a business relationship. Finally, Objectives are statements on the results to be reached and may be applied to the organizations, organizational units or positions.

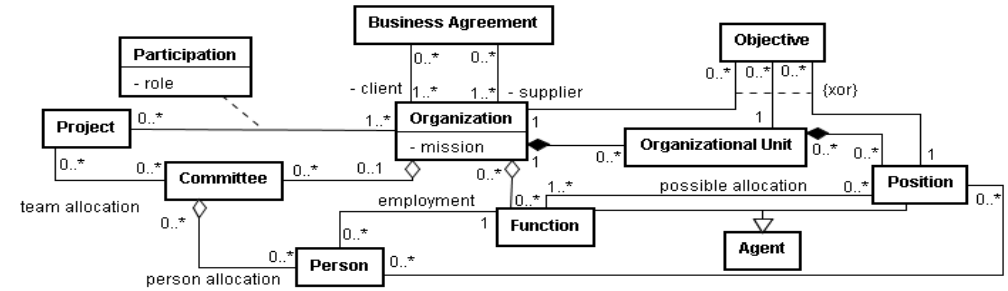

Fig. 2. An SEO Fragment [2].

\section{Reengineering of the Software Enterprise Ontology}

In this section we present a part of the reengineered Software Enterprise Ontology. Due to lack of space, some aspects were not shown here. We tried to include here the most relevant aspects considering the use of UFO.

As mentioned in section 2, UFO defines that agents are capable to perform actions with some intention. The analyzed fragment of SEO (Fig. 2) does not agree with this conceptualization. Since Agent is a generalization of Position and Function, it is better characterized as a Normative Description. So, we modified the original term Agent to Profile, as shown in Figure 3. Still considering the concept of agent in UFO, we can say that organizations, organizational units and teams are social agents, whereas people are physical agents. Moreover, agents have intentions expressed by goals. Thus, the concept Intention was included in the new version of SEO, where an intention is the purpose which actions are planned and performed for, and Goal is the propositional content of an intention [5]. The main intention of a social agent is its Mission. In order to maintain the alignment with the terms used in UFO, we replaced the term Objective by Goal. Furthermore, the perception that a team is also an agent drove us to the conceptualization that teams also have intentions and goals. Otherwise, the perception that a position is a normative description showed that it does not make any sense to associate goals to a position.

In the original version of SEO, an organization is defined as "an organized group of people working together for the fulfillment of a mission". However, people are kinds and they exist independently of organizations. In fact, people start playing the role of Human Resource within an organization when they are employed in it. So, an Organization is better characterized as a social agent which employs human resources for performing actions to achieve its goals.

Organizations can be divided into organizational units. An Organizational Unit can be defined as a grouping of human resources (the human resources allotted in it), goals and intentions, established according to the content homogeneity and alignment to the organization's goals. Similarly, a Team (term used as a substitute for the term 
Committee from the original version) is a grouping of human resources established with a specific purpose.

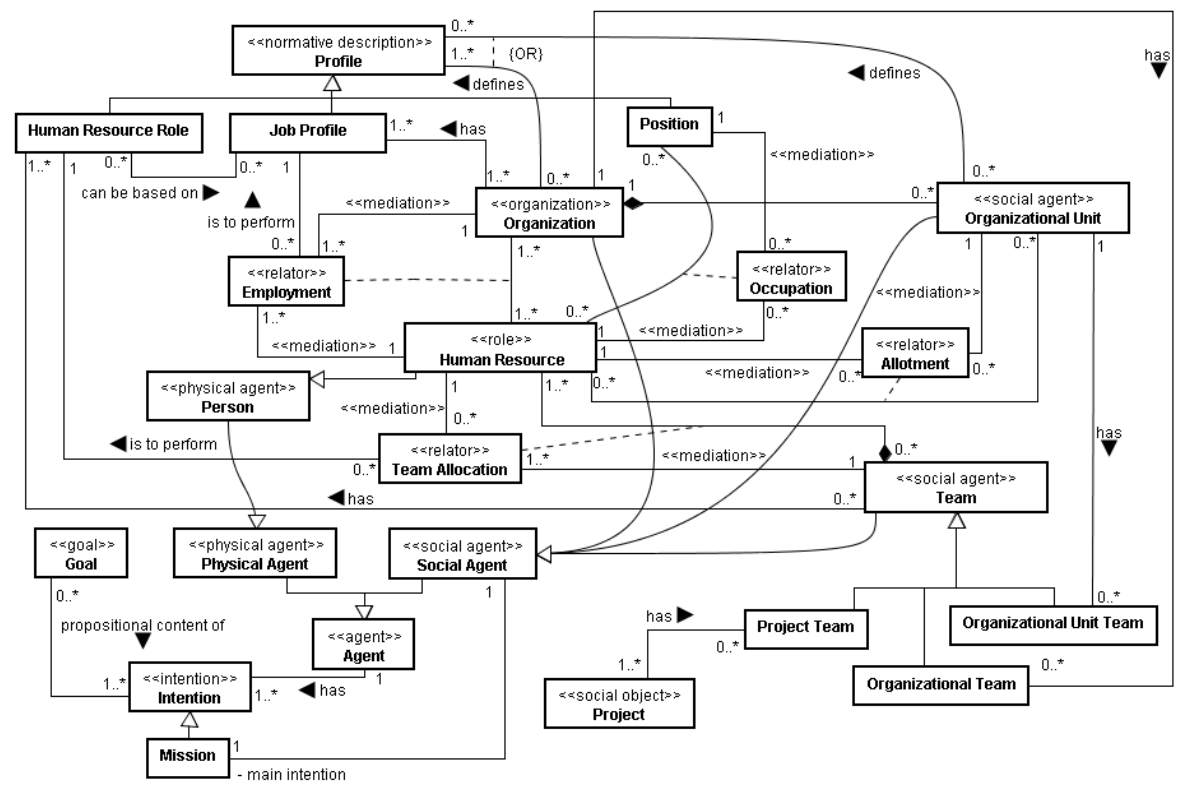

Fig. 3. Fragment of the new version of SEO that includes Agents and Human Resources.

In the original version of SEO, an objective could be exclusively of one (and only one) organization or organizational unit. Analyzing this relation from the UFO perspective, we noticed that the singleness and exclusivity restrictions were not covering some situations. In the real-world it is possible, for example, that an organization has the goal of "decreasing $10 \%$ product defect ratio" and this would be also a goal of some (or all) of its organizational units. Then, those restrictions were abandoned. In the new version, despite an intention is inherent to only one agent, it is possible to get different intentions when goals are the same. In other words, one goal can be the propositional content of intentions of different agents.

Turning the discussion to the concept of team, with the new conceptions that were adopted, we concluded that the original model was not appropriate when it determined that teams (committees) are parts of organizations and can be allocated to projects. Thus, in the new vision, a team can be established to fulfill a purpose in the context of a project, an organization or an organizational unit, being, respectively, a Project Team, an Organizational Team or an Organizational Unit Team.

As it was said before, people play the role of human resources of an organization when they are employed in it. The relation "employment" between Organization and Human Resource is a material relation ${ }^{5}$ universal and, therefore, there is a relator universal (Employment) whose instances are individuals capable of connecting instances of both these entities. This relator is directly related to the record of the

${ }^{5}$ Material relations have material structure on their own, and their relata are mediated by individuals that are called relators [3]. 
events, which establish the employment of a person in an organization, and thus it has as properties, among others, the 'start date' and 'finish date' of the employment. Moreover, this relator is associated with Job Profile, indicating that a human resource fulfils a job profile when it is employed in an organization (e.g., a human resource employed in the job profile of a system analyst in an organization).

Similar situations happen with the other relations of the Human Resource role, namely Team Allocation, Occupation and Allotment. The first one records the occurrence of the event of allocation of a human resource to a team, where it plays a human resource role (e.g., a human resource allocated as a project manager in a project team). The second refers to the occupation of a position by a human resource (e.g., a human resource occupying the position of portfolio manager). At last, the relator Allotment records the event of allotting a human resource in an organizational unit (e. g., a human resource allotted in the development systems unit). Job Profile, Human Resource Role and Position are descriptions of profiles that are needed for acting in specific contexts, as said before, and are, therefore, normative descriptions.

The creation of these relators is an important change in SEO, driven by the use of UFO. These concepts were included because they not only connect other entities, but also define a set of characteristics of the relationship owners (and not do so to the connected entities), allowing better coverage of real-world aspects, such as the perception that all these relators record events (broadly speaking, a relator can be seen as a static representation of an event) and, consequently, they have temporal properties. For example, an employment, despite of being represented statically, fundamentally deals with an event that has a beginning and an end, and it involves a human resource occupying a job profile in an organization. Do not representing employment as a concept (as was done in the original version of the SEO) would not allow the identification of crucial information, such as when the employment began and when it was finished. Furthermore, in the original version of SEO, a person occupies only a job profile and, in fact, an employment is to perform a job profile, but a human resource can occupy several job profiles, since it can have several employments, as it is modeled in the new version of SEO.

Analogous reflection can be done for Team Allocation, Allotment and Occupation. Moreover, it is possible to identify that a team allocation involves a human resource playing a human resource role in the team, what allows that the same human resource to be allocated to several roles in the same team (e.g., a human resource can play the designer and programmer roles in the same project team). In the original version of SEO, a person is allocated to a team without identifying her role. Consequently, it was not possible either to identify her specific functions and responsibilities in the team, nor to allocate her to different roles in the same team.

During the reengineering of SEO, several restrictions were identified and, since the models did not capture several of them, we defined axioms to make them explicit. For instance, the following axiom holds: if a human resource $h r$ occupies, in a time window $[t 1, t 2]$, a position $p$ in an organization $\mathrm{org}$, then this time window must be contained in the time window $[t 3, t 4]$ in which $h r$ is employed in org.

( $\forall$ hr $\in$ Human Resource, $p \in$ Position, org $\in$ Organization) (occupation ( $h r, p, t 1, t 2) \wedge$ defines $($ org, $p)) \rightarrow$ employment $(h r, o r g, t 3, t 4) \wedge(t 1 \geq t 3 \wedge t 2 \leq t 4)$

Figure 4 presents the fragment of the new version of the SEO that deals with projects. 


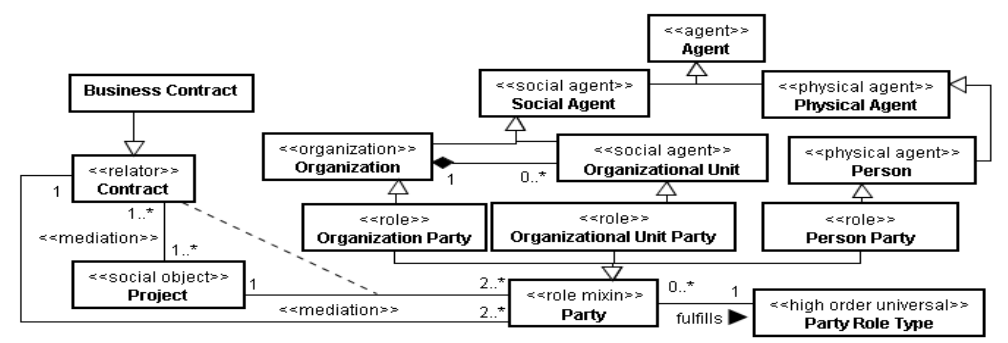

Fig. 4. Fragment of the new version of the SEO that deals with projects.

In the original version of the SEO, the participation of organizations in projects was represented by the relation Participation, which has the property role that indicates the kind of their participation. Moreover, there was the concept of Business Agreement that is defined as an agreement between two or more organizations (clients and suppliers), as shown in Fig. 2. This model has several problems. First, it allows that a project has only one participation, what does not seem to make sense in reality. Projects typically involve at least two parties, playing different roles. Second, the model is negligent concerning the participation of the same organization by different ways in the same project. Can the same organization participate playing different roles? Third, only organizations can participate in projects, making it impossible to represent situations in which a person contracts (or is contracted to develop) a project, nor situations in which an organizational unit contracts or is contracted in a project context. Fourth, there is no relationship between projects and business agreement. Moreover, business agreements have two types of roles predefined: client and supplier, which do not seem to be related with the roles defined in participations.

Trying to solve these problems, in the new version of SEO, the participation on projects and the business agreement were treated by the introduction of the concepts Party and Contract. Party is a form of acting in a project that may be carried out by organizations, organizational units or people. A contract is an agreement established between parties. When a contract has business features, it is a Business Contract.

Analyzing the concept Party at the light of UFO, we can say that it is a role that can be played by different and disjunctive kinds. In other words, Party is a role mixin. According to [3], modeling situations like that has been a recurring problem on the literature. Fig. 5 presents two ontologically incorrect models that could be proposed, if important ontological distinctions of the UFO would not be taken into account.

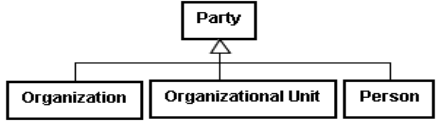

(a)

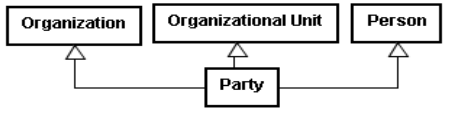

(b)

Fig. 5. Ontologically incorrect models.

In Fig. 5(a), the role Party is defined as a super-type of Person, Organization and Organizational Unit. Ontologically this model is not correct, because it assumes that all instances of Person, Organization and Organizational Unit are necessarily parties, what does not occur in the real-world, since a person still is a person even if she is not a party. The same happens with an organization and an organizational unit. 
In Fig. 5(b), the role Party is defined as a subtype of Organization, Organizational Unit and Person. Ontologically this model is neither correct, because it indicates that Party has identity principles that are common to Organization, Organizational Unit and Person, what does not occur in the real-world, since it is not possible that a party is at the same time an organization, an organizational unit and a person.

In order to solve this problem, Guizzardi proposes the pattern illustrated in Fig. 6.

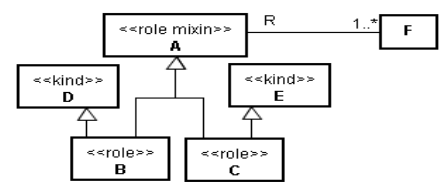

Fig. 6. Pattern for roles with multiple and disjunctive kinds [3].

Applying this pattern to model the concept Party in SEO, Party is a role mixin and, thus, does not have direct instances and includes different types of roles: Organization Party, Organizational Unit Party and Person Party. These roles are disjunctive. For example, the organization $O$ can be an instance of Organization Party, what means that, in a given moment, $O$ plays the role of Organization Party. Organization, Organizational Unit and Person are the substantial sortals that supply the identity principles to instances of Organization Party, Organizational Unit Party and Person Party, respectively. Party is relationally dependent on Project, since it is a role that an organization, organizational unit or person can play in a project context.

Finally, for solving the problem of roles in business agreement and roles in projects, we introduced the concept of Party Role Type that, as it is pointed out by its name, identifies the role that some parties play. Party Role Type is a high order universal, so its instances are first order universals, such as Client, Supplier and Partner. The singleness of Party to Project and Party to Party Role Type allows the participation of the same agent (an organization, an organizational unit or a person) as different parties in contracts of the same project. Moreover, we established that a project has at least two parties, as it is shown in Fig. 4.

\section{Related Works}

Some works that deal with evaluation and improvement of conceptual models based on ontological foundations have been developed in the last years. Guarino and Welty [7] developed the OntoClean methodology, which aims to provide guidance on which kinds of ontological decisions need to be made, and on how these decisions can be evaluated based on general ontological notions drawn from philosophical ontology. In [8] Welty et al. report the results of experiments that measure the advantages achieved from the use of ontologies improved based on OntoClean. Finally, Silva et al. [9] applied a technique that is based on OntoClean, called VERONTO (ONTOlogical VERification) to improve analysis patterns in the geographic domain.

As OntoClean, UFO is being used to evaluate, re-design and give real-world semantics to domain ontologies [5]. Moreover, UFO makes other distinctions and provides more guidelines to evaluate conceptual models than OntoClean does. 
Considering the use of UFO for this purpose, Guizzardi et al. [5] reengineered a Software Process Ontology, while Falbo and Nardi [6] evolved a Software Requirements Ontology. In both cases, ontological problems were identified and solved using UFO, such as in this work.

\section{Conclusion}

This paper presented the reengineering of a fragment of the Software Enterprise Ontology (SEO) defined in [2]. The new version of the ontology was obtained by mapping the concepts of its original version to the concepts of the Unified Foundational Ontology (UFO) [3,5]. The use of UFO allowed identifying several problems and driving the reengineering of the ontology, making explicit ontological commitments that were implicit and elucidating conceptual mistakes.

The need for reengineering SEO was identified during works that aimed reusing some of its concepts, what could not be done because there were problems and limitations related to real-world semantics. This situation corroborate, as argued by Guizzardi et al. [5], that the use of the UFO contributes to achieve quality attributes needed for domain ontologies. The changes done in SEO allowed the reuse and integration of it in the context of a measurement ontology which considers high maturity aspects and that is in final phase of building.

\section{References}

[1] Oliveira, K., Zlot, F., Rocha, A. R. et al.: Domain Oriented Software Development Environments. Journal of Systems and Software, 72(2):145-161 (2004).

[2] Villela, K., Rocha, A.R., Travassos, G.H. et al.: The Use of an Enterprise Ontology to Support knowledge Management in Software Development Environments. Journal of the Brazilian Computer Society, Porto Alegre, Brazil, 11(2): 45-59 (2005).

[3] Guizzardi, G.: Ontological Foundations for Structural Conceptual Models. Universal Press, The Netherlands, ISBN 90-75176-81-3 (2005).

[4] Guarino, N.: Formal Ontology and Information Systems. In Proceedings of International Conference in Formal Ontology and Information Systems, pp 3-15 (1998).

[5] Guizzardi, G., Falbo, R. A., Guizzardi, R. S. S.: Grounding Software Domain Ontologies in the Unified Foundational Ontology (UFO): The case of the ODE Software Process Ontology. In Proceedings of the XI Iberoamerican Workshop on Requirements Engineering and Software Environments, pp 244-251 (2008).

[6] Falbo, R. A., Nardi, J. C.: Evolving a Software Requirements Ontology. XXXIV Conferencia Latinoamericana de Informática, Santa Fe, Argentina, pp 300-309 (2008).

[7] Guarino, N., Welty, C.: Evaluating Ontological Decisions with OntoClean. Communications of the ACM, 45(2): 61-65 (2002).

[8] Welty, C., Mahindru, R., Chu-Carroll, J.: Evaluating Ontology Cleaning. In Proceedings of the National Conference on Artificial Intelligence, pp 311-316 (2004).

[9] Silva, E. O., Lisboa, F. J., Oliveira, A. P., Gonçalves, G. S.:Improving Analysis Patterns in the Geographic Domain Using Ontological Meta-properties. In Proceedings of International Conference on Enterprise Information Systems, pp 256-261(2008). 